\title{
Usability of LTE for Transmitting Radar Data from DLR's Research Aircraft DO 228-212
}

\author{
Stefan V. Baumgartner ${ }^{1}$, Daniel Rosigkeit, Anton Nottensteiner ${ }^{1}$ \\ ${ }^{1}$ German Aerospace Center (DLR), Microwaves and Radar Institute, \\ 82234 Oberpfaffenhofen, Germany, \\ stefan.baumgartner@dlr.de
}

\begin{abstract}
:
In the paper the usability of long term evolution (LTE) data transmission from an aircraft to the ground is investigated. Theoretical analyses and experimental measurements have been carried out by using a commercial low-cost LTE modem and the existing LTE base station infrastructure on ground. No hardware modifications were made. During the experiments the aircraft was operated in altitudes of approximately 1800 and $2800 \mathrm{~m}$ above ground. Over wide areas a stable LTE connection was achieved.
\end{abstract}

Key words: LTE, data transmission, aircraft, synthetic aperture radar (SAR), traffic management

\section{Introduction}

The DLR Microwaves and Radar Institute operates the multi-frequency and multi-channel synthetic aperture radar (SAR) sensor F-SAR [1], which is installed onboard a Dornier DO 228-212 research aircraft. Commonly, for most scientific remote sensing applications the raw radar data acquired with this sensor are processed to fully focused SAR images on ground after the aircraft has landed. For processing, the hard disks or solid state disks are removed from the aircraft and brought to the Institute's data processing facility.

Driven by DLR internal projects dedicated to traffic management for large scale events and disasters (e.g., the VABENE++ project [2]), a powerful onboard processor was developed during the past years. Processing of the radar data can now be carried out directly during the flight onboard the aircraft, parallel to data acquisition. The onboard processor has two main objectives:

- Extraction of relevant traffic information from the multi-channel radar data, preferably in real-time: this involves the detection of moving road vehicles and the estimation of their geographical positions, velocities and moving directions with high accuracy

- Generation of fully polarimetric highresolution SAR images which can be used for disaster management (e.g., for infrastructure monitoring, for monitoring of flooded areas, etc.).
For traffic management and disaster monitoring it is essential, that the extracted traffic information (size of a few kByte) and the generated SAR images (size of dozens to hundreds of MBytes) are transferred to a server on ground immediately. For this task a data link is mandatory.

During some test flight campaigns a C-band microwave data link and an experimental optical laser communication link, developed by our colleagues from the DLR Communications and Navigation Institute, were successfully tested. These data links require a dedicated (mobile) ground station with an antenna or telescope which tracks the aircraft precisely. Both data link systems are quite complex, expensive and require some time and man power for setting up the (mobile) ground stations and for keeping them in operation.

One advantage, compared to conventional and even more expensive satellite communication links, is the achievable high data rate which is in the order of $10 \mathrm{Mbit} / \mathrm{s}$ for the microwave link and even larger for the optical link. However, under circumstances it might be difficult to install the ground station close to the event or disaster location within a small timeframe.

An alternative and low-cost data transmission technique, to our knowledge operationally not yet used for transmitting data from aircrafts, is long term evolution (LTE). In recent years several tests where made with LTE in fast moving vehicles. For instance, in a high speed Transrapid train moving with up to $430 \mathrm{~km} / \mathrm{h}$ a 
stable LTE connection with data rates of 36 to $46 \mathrm{Mbit} / \mathrm{s}$ was achieved [3]. Also first airborne experiments with an Airbus A320 and some special not off-the-shelf ground stations, with antennas oriented to the sky, were very promising [4].

The hardware, integration, maintenance and operation costs for LTE are expected to be relatively low in comparison to a dedicated microwave link, which additionally require a dedicated ground station and a time and location dependent operation license.

For our radar-based traffic management applications we want to know whether the existing standard LTE infrastructure of the German Telekom can be used with a commercially available LTE modem for transmitting radar images and traffic data from our research aircraft DO 228-212 to the ground and internet.

In the following sections the considered LTE performance parameters, some simulation results and the measurement results acquired during an airborne experiment are presented and discussed.

\section{LTE Performance Parameters}

Important LTE performance parameters are the transmit $(R X)$ and receive $(R X)$ data rates, the signal-to-interference plus noise ratio (SINR), the received signal strength indicator (RSSI), the reference signal received power (RSRP), and the reference signal received quality (RSRQ) [6]. These parameters are mainly influenced by the transmit power of the LTE user equipment installed in the aircraft, the distance between the LTE base station and the aircraft, and the patterns and tilt angles of the antennas installed in the base stations on ground and in the aircraft.

The RSRP value represents the power contained in one LTE carrier of $15 \mathrm{kHz}$ bandwidth. With the RSRP parameter different cells using the same carrier frequency can be compared in the LTE network and handover decisions can be made.

The RSRP and the closely related RSSI values alone can generally not be used for drawing final conclusions regarding the signal quality, since the influence of noise, intersymbol interferences (ISI) and other disturbing interferences are not reflected by these values. For assessing the signal quality more comprehensively, it is necessary to consider also the SINR and RSRQ values.

In Tab. 1 a classification of different RSRP, SINR and RSRQ values is made (see, e.g., [7]).
Tab. 1: Quality and usability of the LTE signal.

\begin{tabular}{|c|l|l|c|c|}
\hline $\begin{array}{c}\text { RSRP } \\
\text { [dBm] }\end{array}$ & $\begin{array}{c}\text { SINR } \\
\text { [dB] }\end{array}$ & $\begin{array}{c}\text { RSRQ } \\
\text { [dB] }\end{array}$ & $\begin{array}{c}\text { Receiving } \\
\text { Quality }\end{array}$ & $\begin{array}{c}\text { Signal } \\
\text { Usability }\end{array}$ \\
\hline $\begin{array}{c}>-70 \text { to } \\
-79\end{array}$ & $>10$ & $\begin{array}{l}-3 \text { to } \\
-5\end{array}$ & $\begin{array}{c}\text { very good } \\
\text { level }\end{array}$ & $\begin{array}{c}\text { VolP, very } \\
\text { high data } \\
\text { rate }\end{array}$ \\
\hline $\begin{array}{c}-80 \text { to } \\
-89\end{array}$ & $\begin{array}{l}6 \text { to } \\
10\end{array}$ & $\begin{array}{l}-6 \text { to } \\
-8\end{array}$ & good level & $\begin{array}{c}\text { high data } \\
\text { rate }\end{array}$ \\
\hline-90 to & 0 to & -9 to & average & half data \\
-100 & 5 & -15 & level & rate \\
\hline $\begin{array}{c}-101 \text { to } \\
-110\end{array}$ & $<0$ & $\begin{array}{l}-16 \text { to } \\
-20\end{array}$ & $\begin{array}{c}\text { poor } \\
\text { signal }\end{array}$ & $\begin{array}{c}\text { non stable } \\
\text { connection }\end{array}$ \\
\hline
\end{tabular}

Tab. 1 suggests that a qualitative statement regarding receiving quality and usability of the LTE signal can be made if either the RSRP, the SINR or the RSRQ value is known.

\section{Simulation}

Before conducting any expensive flight experiments it is interesting to know, if at least theoretically a signal reception onboard the aircraft is possible and if the legally fixed and comparatively low TX power of the LTE user equipment is sufficient to transmit data successfully to the ground station.

For a very rough theoretical performance assessment it is sufficient to compute the expected RSRP values. The RSRP corresponds approximately to the received power $P_{\mathrm{RX}}$ in one channel which can be computed as

$$
P_{\mathrm{RX}}=\frac{P_{\mathrm{TX}} \cdot G_{\mathrm{TX}} \cdot A_{\mathrm{RX}}}{4 \cdot \pi \cdot r^{2} \cdot L}
$$

where $P_{\mathrm{TX}}$ is the transmitted power in one channel, $G_{\mathrm{TX}}$ is the transmit antenna gain, $A_{\mathrm{RX}}$ is the effective area of the receiving antenna, $r$ is the distance between the TX and RX antenna, and $L$ are the losses.

The effective antenna area $A_{\mathrm{RX}}$ can also be expressed as a function of the elevation dependent $R X$ antenna gain

$$
A_{\mathrm{RX}}=\frac{\lambda^{2} \cdot G_{\mathrm{RX}}\left(\theta_{\mathrm{el}}\right)}{4 \cdot \pi}
$$

where $\theta_{\mathrm{el}}$ is the elevation angle of the antenna an $\lambda$ is the wavelength of the signal.

Generally, the antenna main lobes of the LTE base stations are directed towards to the Earth with tilt angels between $0^{\circ}$ and $-10^{\circ}$. Therefore, the signals which will be received in the aircraft may originate from the side lobes and from reflections from the ground. For the theoretical analyses and simulations we have ignored potential reflections from the ground.

In Fig. 1 the power $P_{\mathrm{RX}}$ received at the base station is plotted for two different aircraft 
altitudes of $2000 \mathrm{~m}$ (in blue color) and $3000 \mathrm{~m}$ (black) above ground. Since the exact pattern of the base station antenna was not available, for simplification an ideally SINC shaped elevation antenna pattern without any tapering was considered in the simulation. For the antenna mounted at the aircraft an antenna gain of $0 \mathrm{dBi}$ and a TX power $P_{\mathrm{TX}}$ of $200 \mathrm{~mW}$ (= $23 \mathrm{dBm}$ ) was assumed. With $0 \mathrm{dBi}$ antenna gain also the equivalent intrinsic radiated power $E I R P=P_{\mathrm{TX}} \cdot G_{\mathrm{TX}}$ corresponds to the same value of $23 \mathrm{dBm}$, which is just the maximum EIRP allowed by law in Germany for LTE. For the simulation no additional losses were considered (i.e., $L=0 \mathrm{~dB}$ ) and a $\mathrm{RX}$ antenna tilt angle of $-10^{\circ}$ was assumed.

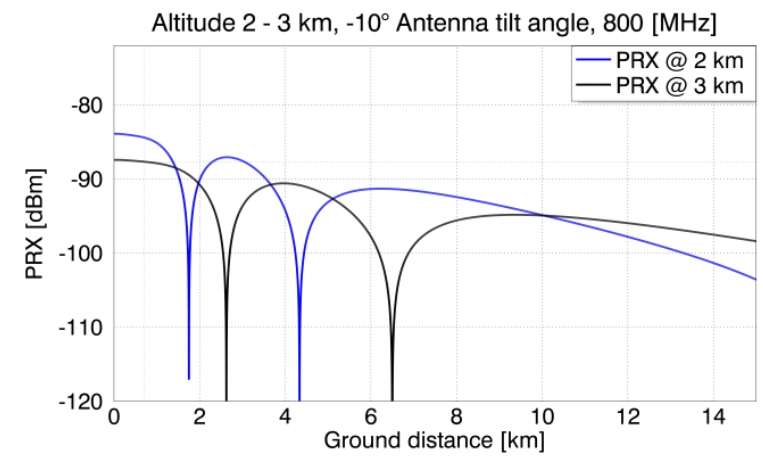

Fig. 1. Expected receive power $P_{R X}$ as a function of ground distance for an aircraft altitude of $2 \mathrm{~km}$ (blue color) and $3 \mathrm{~km}$ (black) above ground.

By comparing the simulation results in Fig. 1 with the RSRP values listed in Tab. 1, at least an average receiving quality level with half data rate can be expected, since the $\mathrm{RX}$ power is larger than $-100 \mathrm{dBm}$ over a wide range. The antenna pattern notches in Fig. 1 are practically less relevant since we expect, as already mentioned, to receive also signals from ground reflections which might be much larger.

\section{Experimental Results}

The measurement flight was conducted at the end of October 2014. For the experiments a conventional FRITZ!Box 6842 LTE modem with the German Telekom as provider was used. The modem was provisionally installed in the right observation window of the DO 228-212, with a depression angle of approximately 40 degree (cf. Fig. 2 right). No external antennas were used.

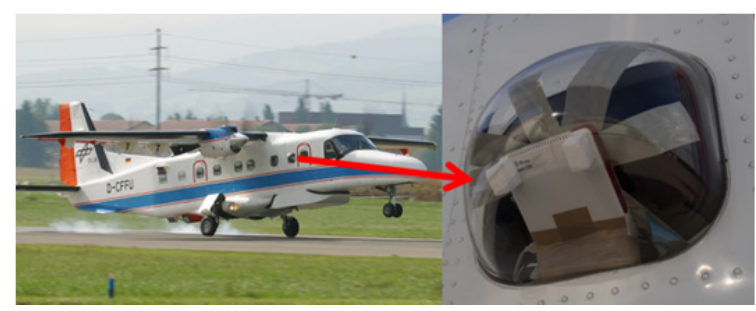

Fig. 2. DLR's DO 228-212 research aircraft (left) and right observation window with provisionally installed LTE FRITZ!Box modem (right).

The LTE performance parameters measured by the FRITZ!Box were logged every two seconds. For logging we have written our own Python script which reads the measurement data directly from the FRITZ!Box's web interface. Additionally to the LTE parameters also the geographical positions of the aircraft were acquired using a conventional GPS tracker.

The flight tracks for carrying out the measurements were carefully planned taking into account the known positions of the LTE base stations on ground. Two laps in different altitudes of $2400 \mathrm{~m}$ and $3350 \mathrm{~m}$ above mean sea level have been flown (these altitudes correspond approximately to altitudes of 1800 and $2800 \mathrm{~m}$ above ground). The measured data corresponding to these altitudes have been spatially synchronized so that a direct LTE performance comparison of the different altitudes is possible.

The LTE base station may transmit simultaneously two signals with different polarizations. The FRITZ!Box has two internal antennas and each of these antennas can receive both transmitted signals. Thus, in total four received signals with different RSRP, SINR and $R S R Q$ values are measured. When the FRITZ!Box is operated in the diversity mode, automatically the signal with the best quality is selected for data reception.

In Fig. 3 the measured maximum RSRP values are plotted (i.e., the maximum of the four RSRP values available at each measurement interval). Missing values indicate that the LTE connection was interrupted. Most of the valid RSRP values are within the -100 to $-80 \mathrm{dBm}$ interval. According to Tab. 1 this interval corresponds to an average to good receiving quality. The RSRP values at $2400 \mathrm{~m}$ altitude are generally better than at $3350 \mathrm{~m}$. 


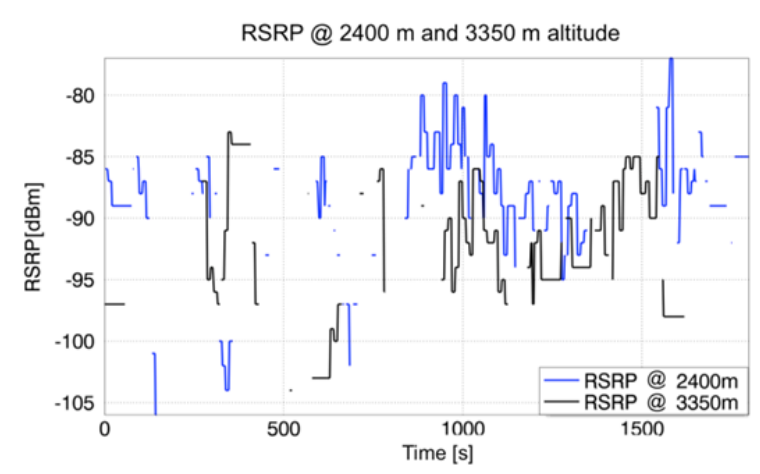

Fig. 3. Measured maximum RSRP values as a function of time for an aircraft altitude of $2400 \mathrm{~m}$ (blue) and $3350 \mathrm{~m}$ (black) above mean sea level.

The measured maximum RSRQ values are plotted in Fig. 4. They behave similar as the RSRP values: for the lower altitude of $2400 \mathrm{~m}$ higher values and, hence, a better LTE performance is observable. Many RSRQ values corresponding to $3350 \mathrm{~m}$ altitude are below -15 $\mathrm{dB}$ which indicates, according to Tab. 1, a poor signal quality and an unstable LTE connection. From this point of view it is recommended to choose the lower altitude for the envisaged traffic monitoring application.

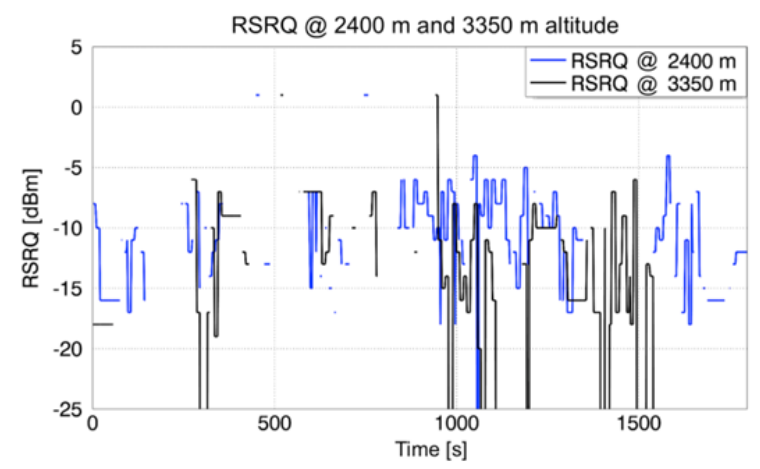

Fig. 4. Measured maximum $R S R Q$ values as a function of time for an aircraft altitude of $2400 \mathrm{~m}$ (blue) and $3350 \mathrm{~m}$ (black) above mean sea level.

The measured maximum SINR values plotted in Fig. 5 are higher for the lower altitude (blue line). Values between 0 and $10 \mathrm{~dB}$ indicate an average to good receiving signal quality with half data rate (cf. Tab. 1). For values below 0 $\mathrm{dB}$, as this is the case for a number of values corresponding to $3350 \mathrm{~m}$ altitude, only a poor signal quality and an unstable LTE connection can be achieved.

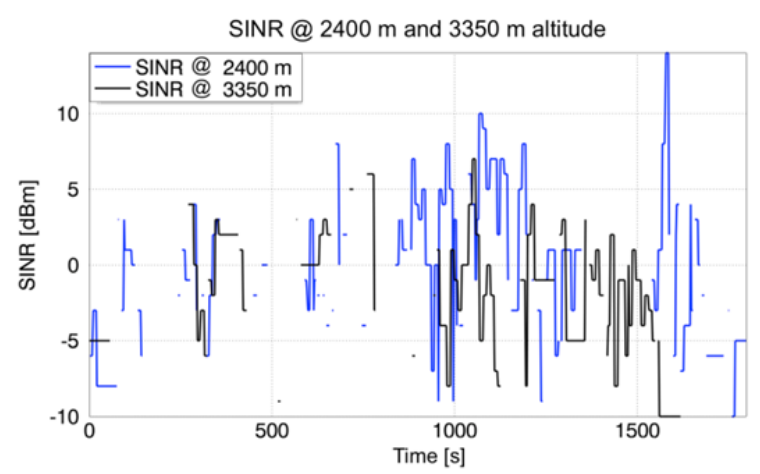

Fig. 5. Measured maximum SINR values as a function of time for an aircraft altitude of $2400 \mathrm{~m}$ (blue) and $3350 \mathrm{~m}$ (black) above mean sea level.

The FRITZ!Box is also able to measure the usable TX and RX data rates. The measured results for an altitude of $2400 \mathrm{~m}$ above mean sea level are depicted in Fig. 6.

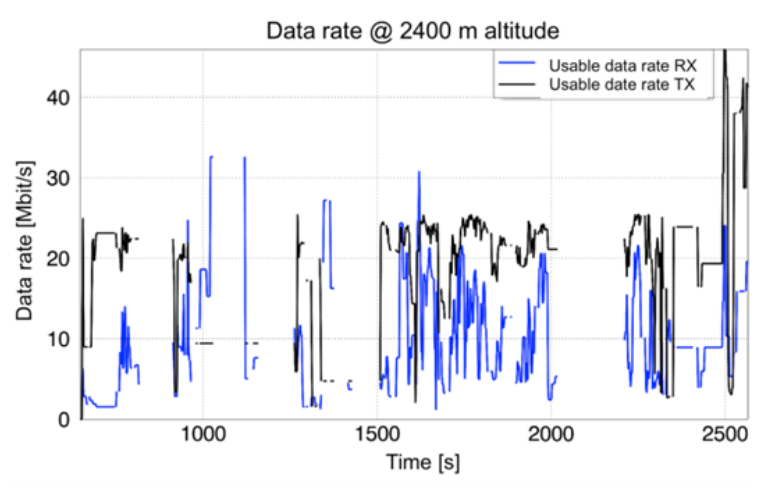

Fig. 6. Measured useable RX (blue) and TX (black) data rates as a function of time for an aircraft altitude of $2400 \mathrm{~m}$ above mean sea level.

Over wide regions the usable data rates are comparable with a home DSL connection. However, a strong fluctuation between approximately 2 and $20 \mathrm{MBit} / \mathrm{s}$ is observable for the $\mathrm{RX}$ case.

The usable TX data rate, which is more in important for our applications, since we want to transmit or "upload" SAR images and traffic data to the ground station, is over wide regions higher than $20 \mathrm{MBit} / \mathrm{s}$.

Although the LTE connection was obviously unstable with several interruptions, it was possible to upload a larger SAR image (cf. Fig. 7) of 270 MByte size to the Dropbox cloud storage without any problems. After interrupts the LTE connection was successfully reestablished and the data transfer automatically resumed. 


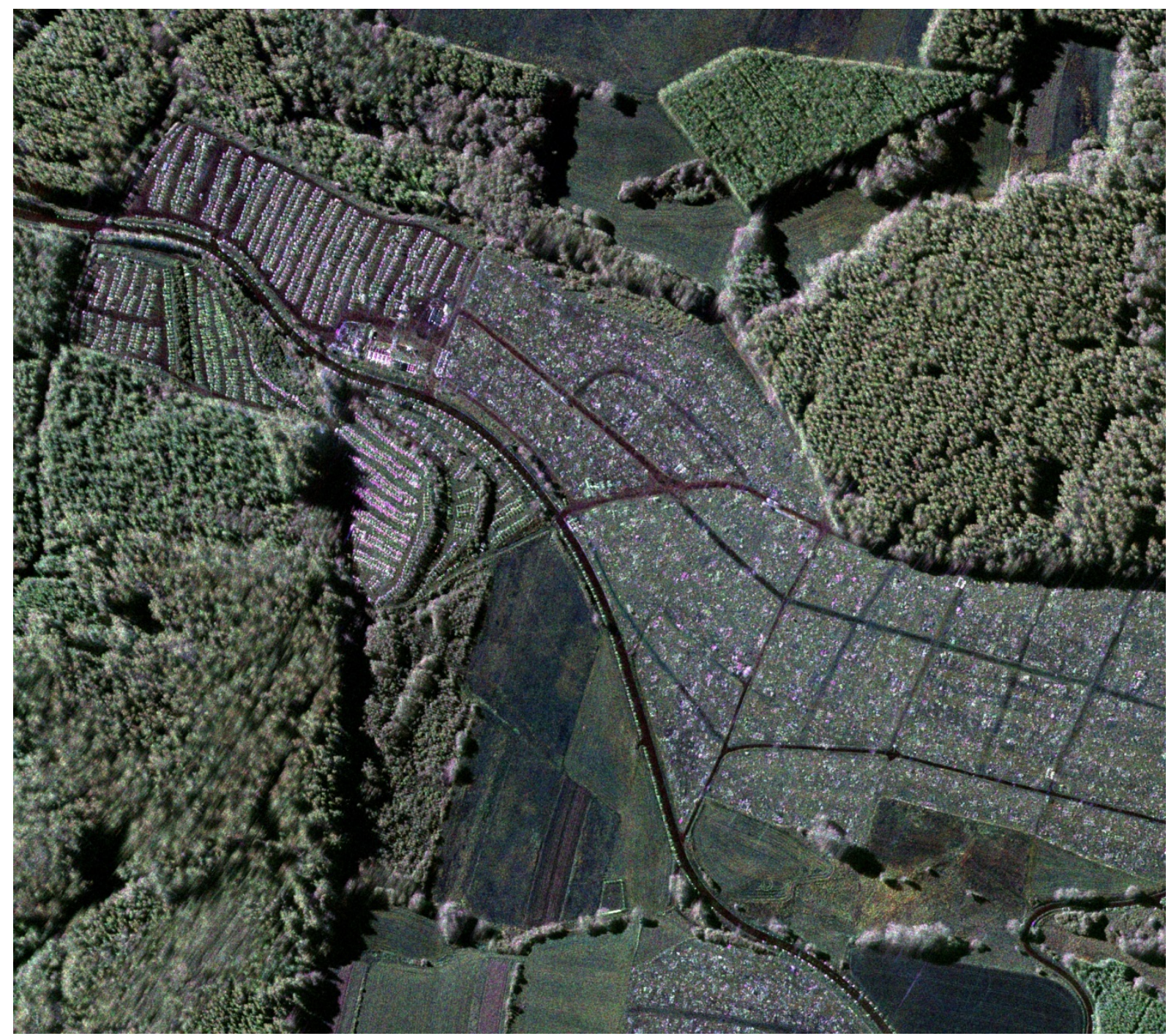

Fig. 7. Fully polarimetric high-resolution SAR image transferred via LTE. The SAR image was acquired four months before the LTE measurement flight during the "Rock am Ring 2014" open air festival. The image has a pixel size of $0.2 \times 0.2 \mathrm{~m}$ and shows a $800 \times 800 \mathrm{~m}$ area containing one of the festival's campsites (from the image center to the bottom right) and parking areas (top left). The colors in the SAR image appear due to different backscatter properties of the objects on the ground.

The transfer of the large SAR image took approximately 14 minutes, which results in an effective average TX data rate of $321 \mathrm{kByte} / \mathrm{s}$. This effective data rate is assumed to be sufficiently high for the envisaged traffic management and disaster monitoring applications.

All measured LTE performance parameters were gelocated by synchronizing the GPS track with the measurements. Keyhole markup language $(\mathrm{KML})$ data files were generated for visualizing the geocoded measurement results with Google Earth.

In Fig. 8 one can see in blue the whole flight track in 2400 and $3350 \mathrm{~m}$ altitude above mean sea level. The vertical colored lines, with a circle at the top, are the measured maximum
RSRP values for a flight altitude of $3350 \mathrm{~m}$. The length of the lines as well as the line and circle color represents the measured RSRP values. Green corresponds to a good signal $(\geq-70$ $\mathrm{dBm})$ and red to poor signal strength $(\leq-110$ $\mathrm{dBm})$.

The green pins are the known locations of the LTE base stations. They were found with the help of an online available net coverage map. Each LTE base station was visited prior to the flight to ensure that it really exists and to acquire important data for later evaluation, e.g., the eNodeB IDs of the stations. The colored circles around the base stations correspond to the broadcast radius on ground, where green is a radius of up to $6 \mathrm{~km}$, yellow up to 9 and red up to $15 \mathrm{~km}$. The aim of the flight track planning 


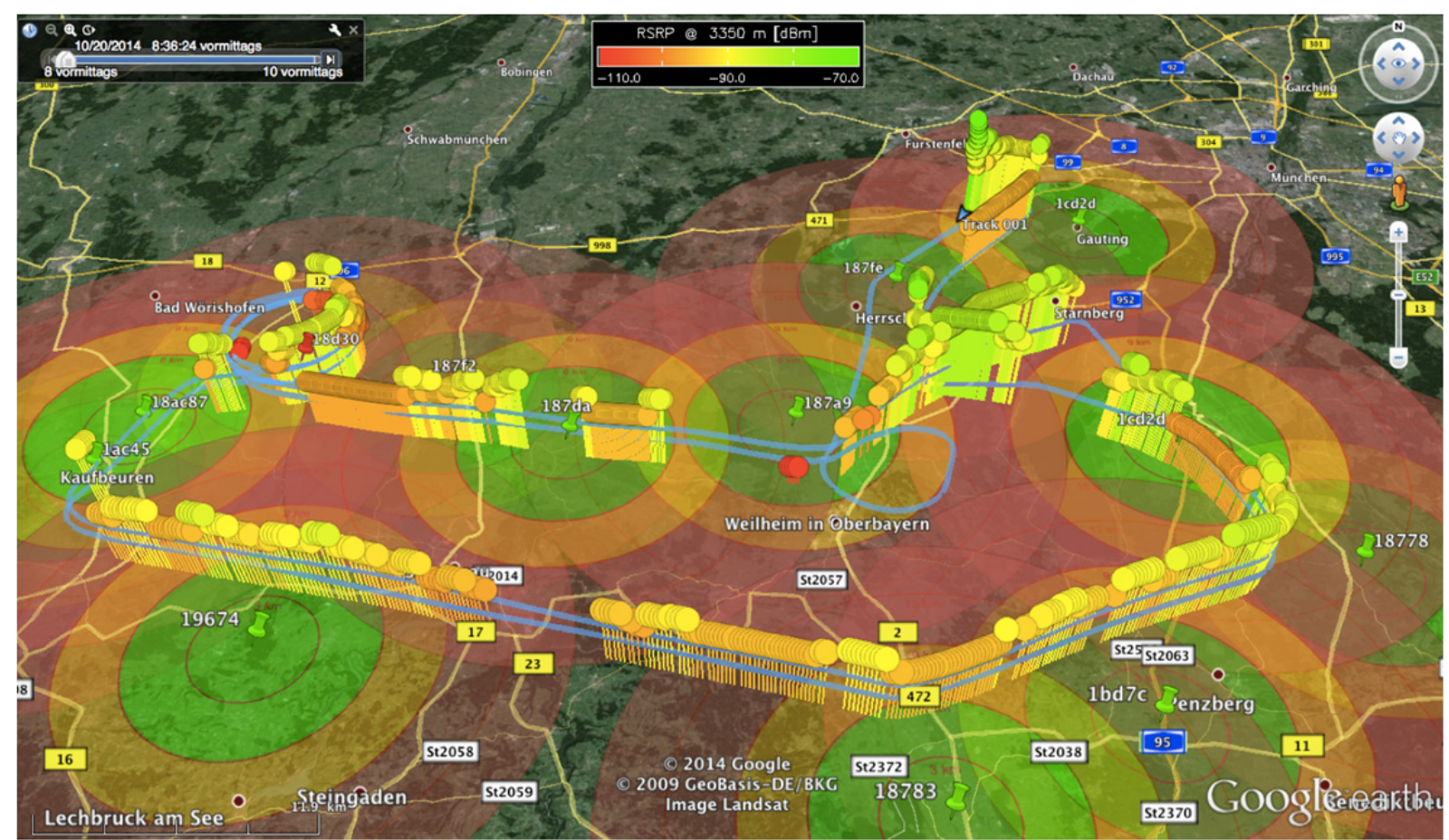

Fig. 8. Geocoded maximum measured RSRP values (colored circles with vertical lines) for $3350 \mathrm{~m}$ altitude above mean sea level. The blue lines represent the complete flight track at 3350 as well as at $2400 \mathrm{~m}$ altitude, the green pins represent the LTE base stations and the colored circles around the pins the distance.

was to ensure that the horizontal distance between the base station and the aircraft as far as possible is not larger than $10 \mathrm{~km}$. According to Fig. 1 this would ensure that the RSRP values are larger than $-95 \mathrm{dBm}$ if the practically less relevant notches caused by the base station antenna pattern are neglected.

The gaps in the measured RSRP data shown in Fig. 8 occur mainly half way between two LTE base stations and directly above the base stations. This behavior was expected since the used FRITZ!Box modem has no omnidirectional but a directed antenna pattern pointing only to the right side with respect to the flight path with the mentioned depression angle of approximately $40^{\circ}$. For a fixed and approved integration of a LTE modem into the DO 228212 research aircraft it is therefore recommended to use at least two external omnidirectional antennas mounted outside the aircraft at the bottom of the fuselage.

\section{Conclusions and Recommendations}

The simulation and measurements show that LTE for radar image and traffic data transmission in principle works in an aircraft flying at an altitude of 2000 to $3000 \mathrm{~m}$ above ground. Many of the measurement gaps, which correspond to a loss of LTE connection, could probably be avoided by using external omnidirectional antennas mounted outside the aircraft at the bottom of the fuselage. In this way negative shading effects caused by the aircraft fuselage and pointing errors of the internal antennas of the FRITZ!Box modem, which was provisionally mounted in the right observation window of the aircraft, can be avoided or at least strongly attenuated. Owing to the provisional experimental setup it was not possible to receive signals coming from the left side or directly from below the aircraft.

For an operational use it is also recommended to take into account the locations of the LTE base stations for the planning of the flight tracks. Especially for transferring large radar images with a size of hundreds of megabytes, the transmission time could be significantly decreased by a proper flight track selection. For the transmission of traffic data with a size of only a few KByte the maximum achievable data rates are of less importance. Here the objective is to keep the gaps where the LTE connection is interrupted as short as possible, so that realtime requirements can be fulfilled.

Furthermore, it is recommended to use data transmission software which supports automatic transmission pause and resume when the LTE connection is lost and re-established.

LTE seems to be a future-proof technology. The LTE infrastructure in Germany and Europe is constantly evolving. Inmarsat and Deutsche Telekom have created the European Aviation 
Network (EAN) in the frame of a partnership. The goal of the EAN is that passengers in aircrafts flying European routes shall have access the high quality and high-speed broadband services [8]. Below altitudes of $10000 \mathrm{ft}$ the internet connection is established via LTE, above $10000 \mathrm{ft}$ via a satellite link. In the frame of the EAN the Deutsche Telekom will build and manage a new powerful mobile broadband network of approximately 300 LTE sites.

\section{References}

[1] A. Reigber, R. Scheiber, M. Jäger, P. PratsIraola, I. Hajnsek, T. Jagdhuber, K.P.

Papathanassiou, M. Nannini, E. Aguilera, S. Baumgartner, R. Horn, A. Nottensteiner, and A. Moreira, Very-High Resolution Airborne Synthetic Aperture Radar Imaging: Signal Processing and Applications, Proceedings of the IEEE, 101 (3), 759-783 (2013); doi: 10.1109/JPROC.2012.2220511

[2] (2016) VABENE++ Traffic Management for Large Scale Events and Disasters [ONLINE]. Available: http://www.dlr.de/vabene/en
[3] (2016) Test LTE im Transrapid [ONLINE]. Available: http://www.silicon.de/41532605/testIte-im-transrapid

[4] (2016) Telekom, Alcatel-Lucent und Airbus testen LTE im Flugzeug [ONLINE]. Available: http://www.areamobile.de/news/21185-telekomalcatel-lucent-und-airbus-testen-Ite-im-flugzeug

[5] S. V. Baumgartner, G. Krieger, Fast GMTI Algorithm For Traffic Monitoring Based On A Priori Knowledge, IEEE Transactions and Geoscience and Remote Sensing, 50 (11), 46264641 (2012); doi: 10.1109/TGRS.2012.2193133

[6] Martin Sauter, From GSM to LTE-Advanced: An Inroduction to Mobile Networks and Mobile Bordband, Wiley, 1st ed. (2011), ISBN 1118861957

[7] (2016) FTS: Was sind RSRP, RSRQ, RSSI und SINR? [ONLINE]. Available: http://blog.antennendiscount24.de/antennen/lteantennen/lte-leistung

[8] (2016) European Aviation Network [ONLINE]. Available: http://www.inmarsat.com/europeanaviation-network 